ditions de préparation du yoghourt, ou peut-être dans les propriétés du lait utilisé, pour modifier les résultats obtenus.

Il n'en apparaît pas moins, d'après nos expériences, que le yoghourt demeure une source excellente de phosphore et de calcium, supérieure aux fromages du point de vue quantitatif, et probablement comparable à eux du point de vue qualificatif : nous rappelons en effet que, d'après nos recherches antérieures [4], l'utilisation physiologique du phosphore et du calcium de fromages tels que le Pont-1'Evêque et le Gruyère est moins élevée que celle du phosphore et $\mathrm{du}$ calcium du lait.

(Laboratoire de Physiologie de la Nutrition de l'Institut National de la Recherche Agronomique et Société scientifique d'Hygiène alimentaire.)

\title{
BIBLIOGRAPHIE
}

[1] H. S. R. Desikachar. et V. Subrahmanyan. Indian Journal Dairy Science, 1948, 1, 123.

[2] Ch. O. Guillaumin. Bull. Soc. Chim. Biol., 1930, 12, 1269; 1932, 14, 105.

[3] W. L. M. Holman. Biochem. Journal, 1943, 37, 256.

[4] L. Randoin, C. Jourdan et J. Causeret. Comples rendus Acad. Agric. $1952,38,161 ; 1952,39,602$.

[5] M. E. Sohultz. Compte rendu du XIII e Congrès Int. Laiterie, 1953, 3, 455 .

[6] A. R. Sundararajan. Indian Journal Med. Res., 1950, 38, 1.

\section{INFLUENCES RESPECTIVES DE LA PROPRETÉ DES USTENSILES ET DU REFROIDISSEIMENT APRÈS LA TRAITE SUR LA QUALITÉ BACTÉRIOLOGIQUE DU LAIT CRU}

\author{
par \\ A. PORTMANN \\ Station Centrale de Microbiologie et Recherches Laitières \\ Jouy-en-Josas (Seine-et-Oise)
}

\section{INTRODUCTION}

Au cours des dernières années, producteurs et professionnels laitiers ont pris conscience de l'extrême importance que présente, en France, l'amélioration de la qualité bactériologique moyenne du lait cru.

Les chiffres du tableau I sont tirés d'une enquête effectuée pendant l'année 1951 et donnent un aperçu de l'état de la qualité bactériologique du lait dans la région parisienne à cette époque. 
TABLEAU I

QUALITÉ BAGTÉRIOLOGIQUE DU LAIT

RECU DANS DIFFERENTES LAITERIES DE LA REGION PARISIENNE EN 1951

\begin{tabular}{|c|c|c|c|c|c|c|}
\hline \multirow[b]{3}{*}{ Mars } & \multirow{3}{*}{$\begin{array}{c}\text { Moyenne de là } \\
\text { température } \\
\text { atmosphérique }\end{array}$} & \multirow{3}{*}{$\begin{array}{c}\begin{array}{c}\text { Nombre } \\
\text { de laiteries }\end{array} \\
1\end{array}$} & \multirow{3}{*}{$\begin{array}{c}\text { Nombre } \\
\text { d'échantillons } \\
\text { de lait examinés }\end{array}$} & \multicolumn{3}{|c|}{$\begin{array}{c}\text { Pourcentage de laits réduisant le } \\
\text { bleu de méthylène en }\end{array}$} \\
\hline & & & & $\begin{array}{l}\text { moins } \\
\text { d'l heure }\end{array}$ & $\left|\begin{array}{c}\text { entre } 1 \text { et } 3 \\
\text { heures }\end{array}\right|$ & $\begin{array}{l}\text { plus de } 3 \\
\text { heures }\end{array}$ \\
\hline & & & & 32 & 15 & 53 \\
\hline Mai à septembre.... & $16^{\circ} 3$ & 5 & 541 & 40 & 32 & 28 \\
\hline
\end{tabular}

On voit que $53 \%$ des laits récoltés en mars et $28 \%$ seulement des laits récoltés pendant les mois d'été réduisaient le bleu de méthylène en plus de trois heures, c'est-à-dire étaient d'une qualité bactériologique que l'on peut qualifier de satisfaisante.

De gros efforts sont faits actuellement par les laiteries pour améliorer la qualité du lait qu'elles reçoivent (primes à la qualité, conseils donnés aux producteurs par les contrôleurs de laiterie), une qualité satisfaisante ne pouvant être obtenue qu'avec le consentement et la participation des producteurs. Ceux-ci disposent de deux moyens pour parvenir au but que l'on se propose, e'est-àdire l'obtention d'un lait cru de bonne qualité bactériologique : l'un de ces moyens réside dans les soins pris pour la récolte du lait et visant à réduire au minimum la contamination initiale; l'autre est représenté par le refroidissement rapide du lait afin de ralentir le développement des microorganismes.

Le rôle respectif de ces deux moyens n'est pas, en général, suffisamment apprécié par le producteur ni même souvent par l'industriel laitier, et les méthodes les plus efficaces et les plus économiques pour obtenir un lait de bonne qualité font encore l'objet de discussions.

C'est ainsi que l'on a souvent tendance à surestimer les avantages que présente l'utilisation du froid artificiel en oubliant que la. qualité bactériologique du lait dépend avant tout des soins qui sont apportés au moment de sa récolte : le froid n'améliore pas la qualité bactériologique du lait, il ne fait que la conserver.

L'étude qui suit a pour but d'apporter quelques précisions sur les données de ce problème en comparant l'influence du nettoyage et de la stérilisation du matériel laitier à celle du refroidissement après la traite.

Dans cette étude, le refroidissement du lait à la ferme était obtenu par l'utilisation de tourniquets hydrauliques. Ces appareils 
que l'on place directement sur les bidons ne nécessitent pas le transvasement du lait et par conséquent, la suppression de cette manipulation diminue les risques de contamination. Ils sont d'un emploi simple, peu coûteux et paraissent les plus susceptibles, actuellement, d'être diffusés dans la pratique [1].

\section{MÉTHODES D'APPRÉCIATION \\ DE LA QUALITE BACTÉRIOLOGIQUE DU LAIT}

\section{Numération des germes totaux.}

Des dilutions convenables du lait à examiner étaient ensemencées en boîtes de Pétri, dans la gélose au lait digéré à la papaïne, les boîtes étaient ensuite incubées à $30^{\circ}$ pendant trois jours.

\section{Réduction du bleu de méthylène.}

Parmi les différentes techniques proposées, nous avons adopté la suivante :

A $20 \mathrm{~cm}^{3}$ du lait à examiner, mesurés aseptiquement dans un tube stérile de $18 \times 180 \mathrm{~mm}$. on ajoute $0 \mathrm{~cm}^{3} 5$ d'une solution stérile de bleu de méthylène (20 mgr. pour $100 \mathrm{~cm}^{3}$ d'eau). Le tube, bouché avec un bouchon de caoutchouc stérile est porté au bain-marie à $37^{\circ}$, retourné toutes les heures et observé toutes les 5 minutes entre 0 et 15 minutes, puis toutes les 15 minutes entre 15 minutes et 3 heures, puis toutes les heures entre 3 heures et 7 heures pour noter le temps de réduction.

\section{Mesure de la conservation du lait.}

Un volume convenable de lait à examiner (habituellement $25 \mathrm{~cm}^{3}$ ) était mis à incuber dans un bain-marie à $23^{\circ}$. Cette température de $23^{\circ}$ a été choisie pour la conservation des échantillons parce que c'est celle qui se rapproche le plus de la moyenne des maxima observés pendant les mois chauds de l'été. Par exemple, pour l'année 1951, dans la région où nous avons effectué nos essais, les températures moyennes atmosphériques sont données dans le tableau II.

TABLEAU II

TEMPÉRATURES ATMOSPHÉRIQUES MOYENNES DURANT L'ÉTÉ 1951

\begin{tabular}{|c|c|c|}
\hline Mois & Températures moyennes & $\begin{array}{l}\text { Moyennes des } \\
\text { températures maxima }\end{array}$ \\
\hline Juin $\ldots \ldots \ldots \ldots \ldots$ & $16^{\circ} 02$ & $19^{\circ} 25$ \\
\hline Juillet. . . . . . . . . & $18^{\circ} 61$ & $25^{\circ} 10$ \\
\hline Août $\ldots \ldots \ldots \ldots \ldots$ & $17 \circ 79$ & $23^{\circ} 15$ \\
\hline Septembre $\ldots \ldots \ldots \ldots$ & $16^{\circ} 79$ & $22^{\circ} 80$ \\
\hline
\end{tabular}


A $23^{\circ}$ le lait se trouve donc placé dans des conditions voisines de celles que l'on rencontre dans la pratique. De ce fait, la flore qui se développe est celle qui a également le plus de chances de se développer chez le consommateur, dans la cuisine ou dans l'office où le pot de lait est conservé.

Le point final de la conservation était observé par deux tests : la coagulation à l'ébullition et la précipitation par l'alcool à $68 \%$. Les techniques utilisées pour ces deux épreuves étaient celles indiquées par Rowllands et al. [2].

a) Coagulation à l'ébullition.

$2 \mathrm{~cm}^{3} \mathrm{du}$ lait à examiner étaient versés dans un tube propre $(16 \times 160 \mathrm{~mm}$.) et portés pendant cinq minutes dans un bain-marie bouillant. On observait la présence ou l'absence de flocons indiquant la coagulation.

b) Coagulation par l'alcool neutre à $68 \%$.

La coagulation du lait était observée après l'addition à $2 \mathrm{~cm}^{3}$ de lait de $2 \mathrm{~cm}^{3}$ d'alcool à $68 \%$ additionné de pourpre de bromocrésol. Ce colorant permet de s'assurer d'abord de la neutralité de l'alcool et d'observer ensuite la teinte que prend le lait après mélange avec le réactif.

\section{PARTIE EXPÉRIMENTALE}

\section{Etude du refroidissement à l'eau courante par tourniquets hydrauliques}

Quatre modèles d'appareils provenant de quatre constructeurs différents ont été étudiés. Ils peuvent en fait se ramener à deux types.

Le type $A$ utilise deux circuits de refroidissement : d'une part ruissellement d'eau sur la paroi externe du bidon et d'autre part circulation d'eau dans trois tubes métalliques verticaux plongeant dans le lait contenu dans le bidon, l'eau arrivant par le tube central et sortant par les deux autres tubes. La pression obtenue à la sortie de l'eau est utilisée pour animer ces tubes d'un mouvement de rotation et permet, de ce fait, d'agiter le lait pendant tout le temps du refroidissement.

Le type $B$ n'utilise que le refroidissement par ruissellement d'eau sur la surface externe du bidon et agitation du lait par une hélice située à l'extrémité d'une tige placée au centre d'un cylindre mû par l'eau sous pression.

a) Vitesse de refroidissement du lait.

Le tableau III donne les températures du lait obtenues après différents temps de fonctionnement pour les deux types d'appareils. 
TABLEAU III

VITESSE DE REFROIDISSEMENT DU LAIT

A L'AIDE DE TOURNIQUETS HYDRAULIQUES

\begin{tabular}{|c|c|c|c|c|}
\hline & \multicolumn{3}{|c|}{ Type A } & Type B \\
\hline & $1^{\text {er }}$ essai & $2^{\theta}$ essai & $3^{e}$ essai & \\
\hline $\begin{array}{l}\text { Température de l'eau à la sortie } \\
\text { du robinet } \ldots \ldots \ldots \ldots \ldots \ldots \ldots\end{array}$ & $14^{\circ}$ & $13^{\circ}$ & $13^{\circ} 5$ & $14^{\circ}$ \\
\hline $\begin{array}{l}\text { Température du lait avant re- } \\
\text { froidissement } \ldots \ldots \ldots \ldots \ldots\end{array}$ & $32^{\circ} 5$ & $34^{\circ}$ & $35^{\circ}$ & $29^{\circ} 5$ \\
\hline $\begin{array}{l}\text { Température du lait après } 10 \\
\text { minutes de fonctionnement } . .\end{array}$ & $15^{\circ} 5$ & $15^{\circ} 5$ & & $19^{\circ}$ \\
\hline $\begin{array}{l}\text { Température du lait après } 15 \\
\text { minutes de fonctionnement .. }\end{array}$ & $14^{\circ} 5$ & $14^{\circ}$ & $14^{\circ} 5$ & 17 \\
\hline $\begin{array}{l}\text { Température du lait après } 20 \\
\text { minutes de fonctionnement . }\end{array}$ & & & & $16^{\circ}$ \\
\hline
\end{tabular}

Avec les appareils utilisés, le type A permet d'obtenir, après une même durée de fonctionnement, un refroidissement plus accentué que le type B.

b) Consommation d'eau.

Elle a été mesurée avec un appareil du type A. Un bidon contenant 20 litres de lait à $33^{\circ}$ était placé sur un support au-dessus d'une bassine de 100 litres destinée à recueillir et mesurer la quantité d'eau traversant l'appareil.

$\mathrm{Au}$ cours de cet essai, on a étudié l'influence du débit d'eau, sur la vitesse de refroidissement du lait et la consommation d'eau (tableau IV).

On voit sur la figure 1 que les courbes de consommation d'eau en fonction de la température atteinte pour des débits de 5 et de 71.5 par minute sont très rapprochées. Dans les deux cas, la quantité d'eau nécessaire pour atteindre $15^{\circ}$ est d'environ 100 litres. La température finale dépend donc surtout de la quantité d'eau utilisée.

La figure 2 (température atteinte en fonction du temps) montre que la vitesse de refroidissement est liée au débit : pour obtenir la température de $16^{\circ}$ dans le lait il faut vingt minutes avec le débit de 5 litres par minute, alors qu'il faut seulement douze minutes environ avec un débit de 71.5 par minute. Toutefois, le débit ne peut varier que dans des limites étroites : en effet, si le débit est 
TABLEAU IV

VITESSE DE REFROIDISSEMENT ET CONSOMMATION D'EAU

\begin{tabular}{|c|c|c|c|c|}
\hline \multirow{2}{*}{$\begin{array}{l}\text { Durée d'utilisation } \\
\text { de l'appareil }\end{array}$} & \multicolumn{2}{|c|}{$\begin{array}{c}\text { Expérience } I \\
\text { Débit de } 5 \text { litres d'eau par minute }\end{array}$} & \multicolumn{2}{|c|}{$\begin{array}{ll} & \text { Expérience } I I \\
\text { Débit de } 71.5 \text { d'eau par minute }\end{array}$} \\
\hline & $\begin{array}{c}\text { Température } \\
\text { du lait } \\
\text { du bidon }\end{array}$ & $\begin{array}{l}\text { Quantité d'eau } \\
\text { utilisée par l'ap. } \\
\text { pareil (en litres) }\end{array}$ & $\begin{array}{c}\text { Température } \\
\text { du lait } \\
\text { du bidon }\end{array}$ & $\begin{array}{l}\text { Quantité d'eau } \\
\text { utilisée par l'ap- } \\
\text { pareil (en litres) }\end{array}$ \\
\hline Début de l'expérience. & $33^{\circ}$ & 0 & $33^{\circ}$ & 0 \\
\hline 5 minutes $\ldots \ldots \ldots \ldots$ & $24^{\circ}$ & 25 & $20^{\circ} 5$ & 37,5 \\
\hline 10 minutes $\ldots \ldots \ldots \ldots$ & $20^{\circ}$ & 50 & $17^{\circ}$ & 75 \\
\hline 15 minutes $\ldots \ldots \ldots \ldots$ & $17 \circ 5$ & 75 & $15^{\circ}$ & 112,5 \\
\hline 20 minutes $\ldots \ldots \ldots \ldots$ & $16^{\circ}$ & 100 & & \\
\hline
\end{tabular}

La température de l'eau à son entrée dans l'appareil était de $14^{\circ}$.

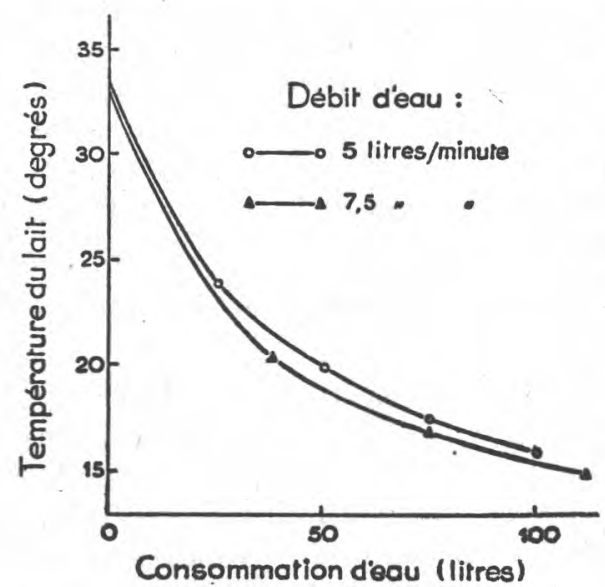

Fig. 1

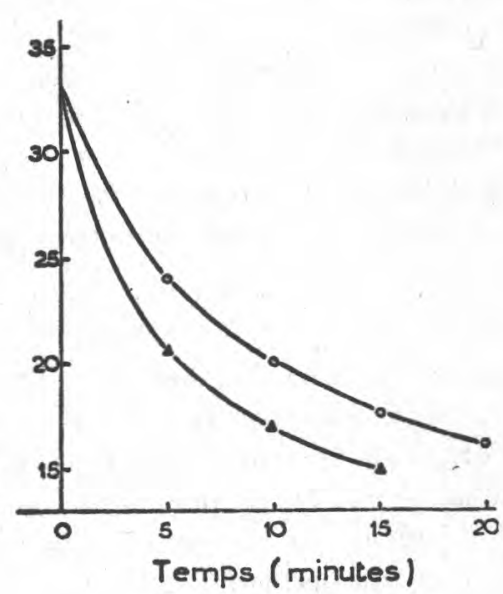

Fig. 2

Refroidissement du lait par des appareils utilisant l'eau courante.

trop faible, la pression d'eau n'est pas suffisante pour faire tourner l'hélice, et si le débit est trop fort, l'eau est mal utilisée en raison de sa mauvaise répartition sur la paroi externe du bidon au cours du ruissellement. 


\section{Influence du refroidissement sur la qualité bactériologique du lait}

\section{A. Refroidissement à $14^{\circ}$ et maintien ultérieur à la température atmosphérique.}

Dans une première série d'essais, la qualité bactériologique du lait refroidi après la traite a été comparée à celle du lait non refroidi.

Pour cela le lait était récolté dans les conditions habituelles de traite, puis le contenu des bidons était mélangé dans une bassine stérile et enfin redistribué dans des bidons stériles. Aussitôt après la répartition en bidons, le lait était refroidi à $14^{\circ}$, à $15^{\circ}$ ou à $16^{\circ}$, sauf celui d'un bidon conservé comme témoin. Le bidon de lait témoin et les bidons de lait refroidi aux diverses températures étaient abandonnés ensuite à la température atmosphérique jusqu'au lendemain (tableau V).

Le lait de la traite du soir, non refroidi et ramassé le lendemain après 17 heures de conservation à la température atmosphérique, coagulait à l'ébullition et était impropre à la pasteurisation; sa qualité bactériologique était très mauvaise (de 35 à plus de 100 millions de germes par centimètre cube).

Le même lait, de qualité bactériologique initiale semblable, refroidi à 14 ou $15^{\circ}$ immédiatement après la traite, et conservé dans les mêmes conditions, ne coagulait à l'ébullition qu'après des temps s'échelonnant, pour les différents essais, entre 5 et 16 heures d'incubation à $23^{\circ}$. Le nombre de germes par centimètre cube était de 2 à 3 millions dans le deuxième essai et de 250.000 à 800.000 dans le troisième essai. La différence observée entre le lait refroidi et non refroidi est done trés significative.

B. Refroidissement à $15^{\circ}, 17^{\circ}$ et $20^{\circ}$ et maintien à cette température jusqu'au lendemain.

Si le refroidissement à $15^{\circ}$ permet d'augmenter la durée de conservation du lait il n'est pas toujours possible d'atteindre cette température avec les tourniquets hydrauliques, l'eau utilisée à la ferme étant parfois à une température trop élevée pour cela.

Dans les essais suivants on a étudié l'influence du refroidissement à une température égale ou supérieure à $15^{\circ}$, le lait étant ensuite maintenu à la température atteinte au moment de l'opération de refroidissement jusqu'au lendemain à $13 \mathrm{~h} .30$.

Lors des deux essais suivants, le lait de la traite du soir était mélangé dans une bassine stérile de 100 litres, puis réparti dans des bidons stériles.

Essais $n^{0} 1$ : Le lait réparti dans trois bidons était refroidi à $15^{\circ}$ dans le premier bidon, à $17^{\circ}$ dans le deuxième et à $20^{\circ}$ dans le 
TABLEAU V

QUALITE BACTERIOLOGIQUE DU LAIT REFROIDI ET NON REFROIDI

\begin{tabular}{|c|c|c|c|c|c|c|c|c|}
\hline & \multicolumn{4}{|c|}{ Température du lait } & \multicolumn{4}{|c|}{ Qualité bactériologique du lait, 16 à 17 heures après la traite } \\
\hline & $\begin{array}{l}\text { Aussitôt } \\
\text { après }\end{array}$ & $\begin{array}{c}\text { Aussitôt } \\
\text { après refroi- }\end{array}$ & $\begin{array}{l}2 \text { heures } \\
\text { après }\end{array}$ & $\begin{array}{c}16 \text { heures } \\
\text { après }\end{array}$ & $\begin{array}{l}\text { Nombre de germes } \\
\text { par } \mathrm{cm}^{3} \text { (milliers) }\end{array}$ & $\begin{array}{c}\text { Temps de réduc- } \\
\text { tion du bleu }\end{array}$ & $\begin{array}{r}\text { Durée de con } \\
\text { avant } \mathrm{c} \\
\end{array}$ & $\begin{array}{l}\text { aservation à } 23^{\circ} \\
\text { oagulation }\end{array}$ \\
\hline & la traite & dissement & la traite & la traite & & de méthylène & par l'alcool & par l'ébullition \\
\hline Essai no 1 & $\begin{array}{l}32^{\circ} 5 \\
33^{\circ} 5 \\
34^{\circ} 5\end{array}$ & $\begin{array}{l}32^{\circ} 5 \\
14^{\circ} \\
15^{\circ}\end{array}$ & $\begin{array}{l}32^{\circ} 5 \\
14^{\circ} \\
15^{\circ}\end{array}$ & $\begin{array}{l}22^{\circ} \\
18^{\circ} \\
17^{\circ}\end{array}$ & & $\begin{array}{l}0 \\
4 \\
4 \mathrm{~h} .\end{array}$ & & $\begin{array}{c}0 \\
16 \mathrm{~h} . \\
16 \mathrm{~h} .\end{array}$ \\
\hline Essai no 2 & $\begin{array}{l}34^{\circ} 5 \\
34^{\circ} \\
34^{\circ}\end{array}$ & $\begin{array}{l}34^{\circ} 5 \\
14^{\circ} \\
15^{\circ}\end{array}$ & & $\begin{array}{l}17^{\circ} \\
15^{\circ} 5 \\
16^{\circ}\end{array}$ & $\begin{array}{r}>100.000 \\
2.080 \\
3.030\end{array}$ & $\begin{array}{l}0 \\
1 \mathrm{~h} . \\
1 \mathrm{~h} .\end{array}$ & $\begin{array}{l}0 \\
8 \mathrm{~h} . \\
7 \mathrm{~h} .\end{array}$ & $\begin{array}{c}0 \\
9 \mathrm{~h} . \\
9 \mathrm{~h} .\end{array}$ \\
\hline Essai $n^{\circ} 3$ & $\begin{array}{l}32^{\circ} \\
32^{\circ} \\
25^{\circ} \\
29^{\circ} 5\end{array}$ & $\begin{array}{l}32^{\circ} \\
14^{\circ} \\
16^{\circ} \\
16^{\circ}\end{array}$ & $\begin{array}{l}28^{\circ} \\
17^{\circ} \\
17^{\circ} \\
17^{\circ}\end{array}$ & $\begin{array}{l}19^{\circ} \\
17^{\circ} 5 \\
17^{\circ} 5 \\
17^{\circ} 5\end{array}$ & $\begin{array}{r}35.000 \\
800 \\
300 \\
250\end{array}$ & $\begin{array}{l}0 \text { h. } 05 \\
1 \text { h. } \\
0 \text { h. } 45 \\
0 \text { h. } 45\end{array}$ & $\begin{array}{l}0 \\
5 \mathrm{~h} . \\
5 \mathrm{~h} . \\
5 \mathrm{~h} .\end{array}$ & $\begin{aligned} & 0 \\
> & 5 \mathrm{~h} . \\
> & 5 \mathrm{~h} . \\
> & 5 \mathrm{~h} .\end{aligned}$ \\
\hline
\end{tabular}


troisième, puis maintenu à ces températures jusqu'au lendemain.

Essais $n^{\circ} 2$ : Le lait était réparti dans deux bidons. Dans le premier, il était refroidi à $15^{\circ}$, puis conservé à cette température jus. qu'au lendemain. Le deuxième bidon était mis dans une salle dont la température était de $6^{\circ}$ pendant $16 \mathrm{~h}$. 30, puis dans une salle à $2{ }^{\circ} 5$ pendant $2 \mathrm{~h}$. 30 , la température du lait était enregistrée pendant toute la durée du refroidissement (tableau VI).

Dans le tableau VI, on voit que pour le lait de l'essai n ${ }^{0} 1$, dont la contamination initiale était très importante $(3,5$ millions de germes par centimètre cube), le refroidissement immédiat à $15^{\circ}$ et la conservation à cette température pendant $19 \mathrm{~h} .30$ étaient tout juste suffisants pour que le lait puisse supporter sans coaguler le chauffage nécessaire à la pasteurisation. Le refroidissement à $17^{\circ}$ et $20^{\circ}$ et la conservation à cette température s'avéraient par contre nettement insuffisants ( 105 à 300 millions de germes par centimètre cube).

Le lait de l'essai no 2 avait une contamination initiale moins importante (200.000 germes au centimètre eube) ; le refroidissement à $15^{\circ}$ et la conservation à la même température pendant 20 heures permettaient d'obtenir du lait ayant une qualité bactériologique meilleure que dans le premier cas (4 millions de germes par centimètre cube). La partie du lait du deuxième essai qui, après la traite, était mise dans une salle dont la température était maintenue à $6^{\circ}$ pendant $16 \mathrm{~h}$. 30 et $2^{\circ} 5$ pendant $2 \mathrm{~h}$. 30 était, après cette conservation, d'une qualité bactériologique ne lui permettant pas de supporter la température de pasteurisation sans coaguler (50 millions de germes par centimètre cube et $21^{\circ}$ Dornic).

En observant la courbe de refroidissement en fonction du temps pour le lait maintenu dans ces dernières conditions (fig. 3), on note qu'il a fallu 7 heures pour que la température s'abaisse de $33^{\circ} 5$ à $16^{\circ}$ et qu'après $5 \mathrm{~h}$. 30 , la température du lait était encore de $20^{\circ}$.

\section{Refroidissement immédiat et différé.}

A la traite du soir (16 h. 30), 80 litres de lait étaient traits en 20 minutes, immédiatement mélangés dans une bassine stérile et répartis dans quatre bidons stériles. Le lait d'un premier bidon était refroidi immédiatement, le second bidon était refroidi 1 heure après la traite, les troisième et quatrième bidons respectivement 2 heures et 3 heures après la traite. Le refroidissement terminé, les bidons étaient abandonnés à la température du laboratoire (230) jusqu'au lendemain à 11 h. 30 , au moment où était effectué le prélèvement des échantillons destinés à l'analyse (tableau VII). 
TABLEAU VI

QUALITÉ BAGTÉRIOLOGIQUE DU LAIT REFROIDI AUSSITOT APRES LA TRAITE

A UNE TEMPERATURE EGALE OU SUPERIEURE A $15^{\circ}$

\begin{tabular}{|c|c|c|c|c|c|c|}
\hline & Lait & $\begin{array}{l}\text { Temps de réduction } \\
\text { du bleu de méthylène }\end{array}$ & $\begin{array}{c}\text { Durée de } \\
\text { avant c } \\
\text { l'alcool }\end{array}$ & $\begin{array}{l}\text { ation à } 23^{\circ} \\
\text { uler par } \\
\text { l'ébullition }\end{array}$ & $\begin{array}{l}\text { Nombre de } \\
\text { germes par } \mathrm{cm}^{3} \\
\text { (milliers) }\end{array}$ & $\begin{array}{c}\text { Acidité } \\
\text { (degrés Dornic) }\end{array}$ \\
\hline Essai n ${ }^{\circ} 1$ & 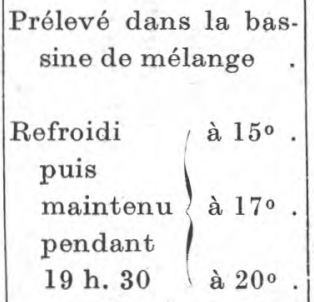 & $\begin{array}{c}2 \mathrm{~h} .45 \\
\text { entre } 0 \text { h. } 15 \text { et } 0 \text { h. } 30 \\
\text { entre } 0 \text { h. } 10 \text { et } 0 \text { h. } 15 \\
0 \text { h. }\end{array}$ & $\begin{array}{l}7 \mathrm{~h} . \\
0 \mathrm{~h} .30 \\
0 \mathrm{~h} . \\
0 \mathrm{~h} .\end{array}$ & $\begin{array}{l}10 \text { h. } 30 \\
2 \text { h. } 45 \\
0 \text { h. } 45 \\
0 \text { h. }\end{array}$ & $\begin{array}{r}3.500 \\
10.000 \\
105.000 \\
300.000\end{array}$ & $\begin{array}{l}19,5 \\
23 \\
35\end{array}$ \\
\hline Essai n ${ }^{\circ} 2$ & $\begin{array}{l}\text { Prélevé dans la bas- } \\
\text { sine de mélange } \ldots \\
\text { Refroidi à } 15^{\circ} \text { pen. } \\
\text { dant } 20 \mathrm{~h} . \ldots \ldots \ldots \\
\text { Conservé dans une } \\
\text { salle à } 6^{\circ} \text { pendant } \\
16 \text { h. } 30 \text { puis à } 2^{\circ} 5 \\
\text { pendant } 2 \text { h. } 30 \ldots\end{array}$ & $\begin{array}{c}3 \mathrm{~h} . \\
0 \mathrm{~h}, 15 \\
\text { entre } 0 \mathrm{~h} .10 \text { et } 0 \mathrm{~h} .15\end{array}$ & $\begin{array}{l}10 \mathrm{~h} .30 \\
1 \mathrm{~h} .\end{array}$ & $\begin{array}{l}13 \text { h. } 30 \\
3 \text { h. } 30 \\
2 \text { h. } 30\end{array}$ & 200 & 17 \\
\hline
\end{tabular}




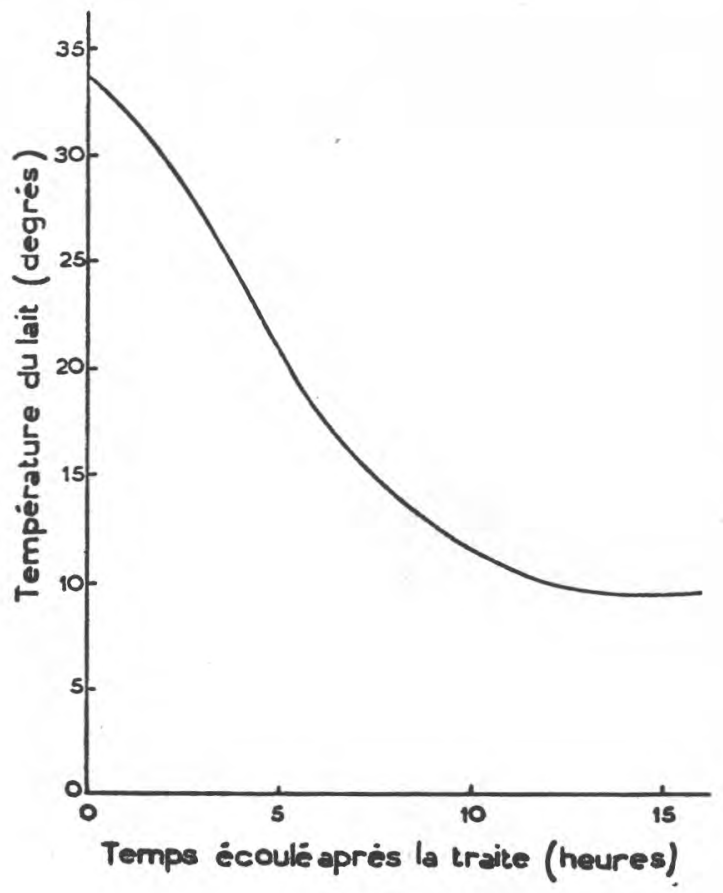

Fig. 3

Evolution de la température du lait contenu dans un bidon placé dans une salle $\grave{a} 6^{\circ}$.

Les refroidissements effectués immédiatement ou 1 heure après la traite permettent d'obtenir une qualité bactériologique sensiblement identique après 19 heures de conservation $(27,5$ et 33 millions de germes par centimètre cube). Par contre, les délais de 2 heures et de 3 heures séparant le moment de la traite du refroidissement ont pour conséquence une augmentation notable du nombre de germes ( 60 et 95 millions par centimètre cube).

Ces résultats, qui montrent la nécessité de refroidir le lait le plus tôt possible après la traite, indiquent également que si le lait est fortement ensemencé dès sa récolte $(300.000$ germes par centimètre cube dans l'expérience) le refroidissement à $15^{\circ}$ est tout juste suffisant pour obtenir une durée de conservation acceptable.

\section{Influence de la propreté de la vaisselle laitière}

Les résultats précédents indiquent déjà que le problème de l'obtention d'un lait peu contaminé dès la traite, reste de la plus grande importance. Or, on sait que la source principale de conta- 


\section{TABLEAU VII}

QUALITE BACTERIOLOGIQUE DU LAIT REFROIDI IMMÉDIATEMENT ET 1 HEURE, 2 HEURES ET 3 HEURES APRËS LA TRAITE

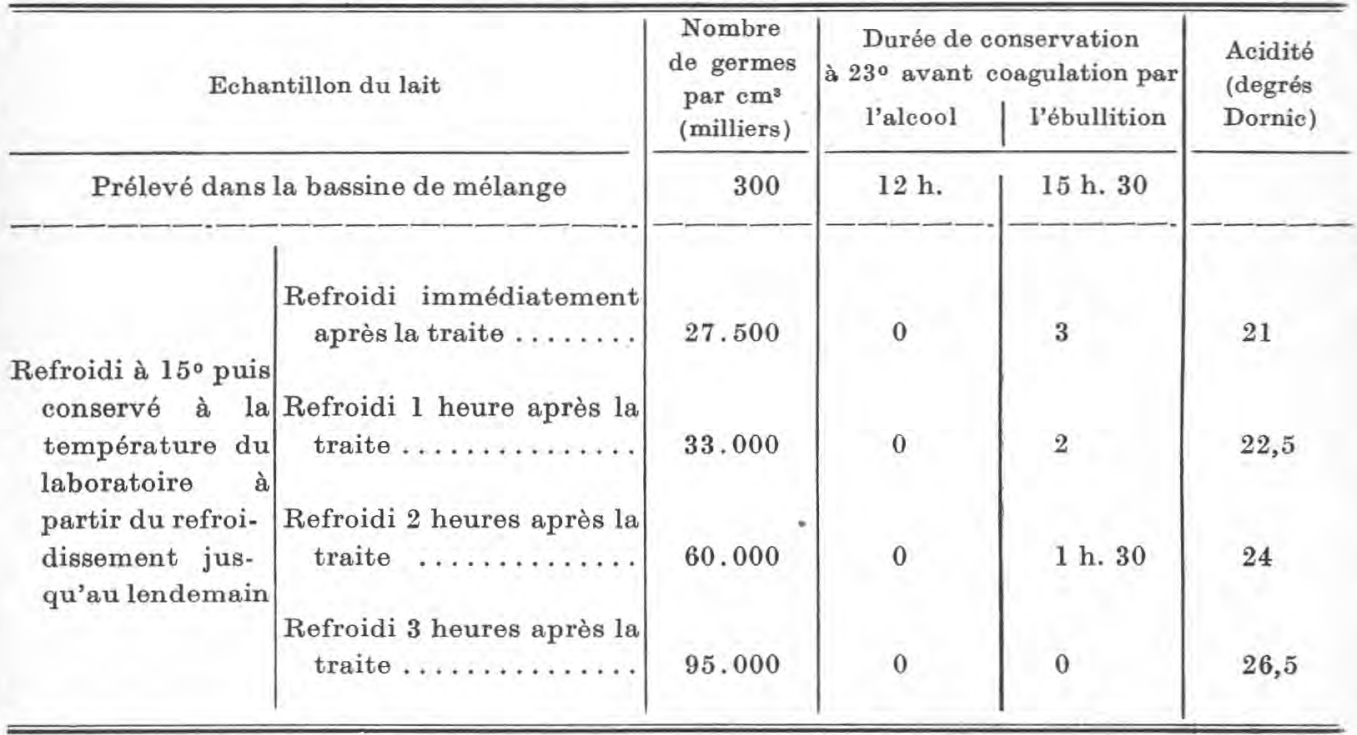

mination provient d'un nettoyage insuffisant de la vaisselle laitière.

Les expériences précédentes ont été effectuées avec le lait obtenu dans les récipients de l'exploitation agricole où avaient lieu nos essais, et sans précautions spéciales pour le nettoyage. Dans la partie qui suit, nous avons comparé l'efficacité du refroidissement sur du lait trait dans du matériel nettoyé avec soin d'une part, et, d'autre part, sur du lait trait dans du matériel nettoyé de façon sommaire, suivant la méthode habituellement utilisée à la ferme où avaient lieu les essais.

Cette ferme située dans le département de l'Yonne représentait assez bien la moyenne des exploitations de la région. La traite était effectuée par le vacher attaché à l'exploitation.

Pour nos essais, nous avons procédé de la façon suivante. Une partie du lait était reçue dans des seaux à traire, filtres et bidons nettoyés à la brosse et à l'eau chaude contenant des cristaux de soudes puis, avant la traite, rinçé avec de l'eau légèrement javellisée. Une deuxième partie était traite dans des seaux, filtres et bidons nettoyés par le personnel de l'exploitation comme cela se faisait d'habitude, par rinçage à l'eau froide et égouttage.

Les résultats indiqués dans le tableau VIII montrent que dans tous les cas où le lait a été trait dans du matériel soigneusement nettoyé et stérilisé, la qualité bactériologique était suffisamment 
TABLEAU VIII

AGTION COMPARÉE DU REFROIDISSEMENT ET DE LA PROPRETE DU LAIT SUR LA QUALITÉ BAGTÉRIOLOGIQUE

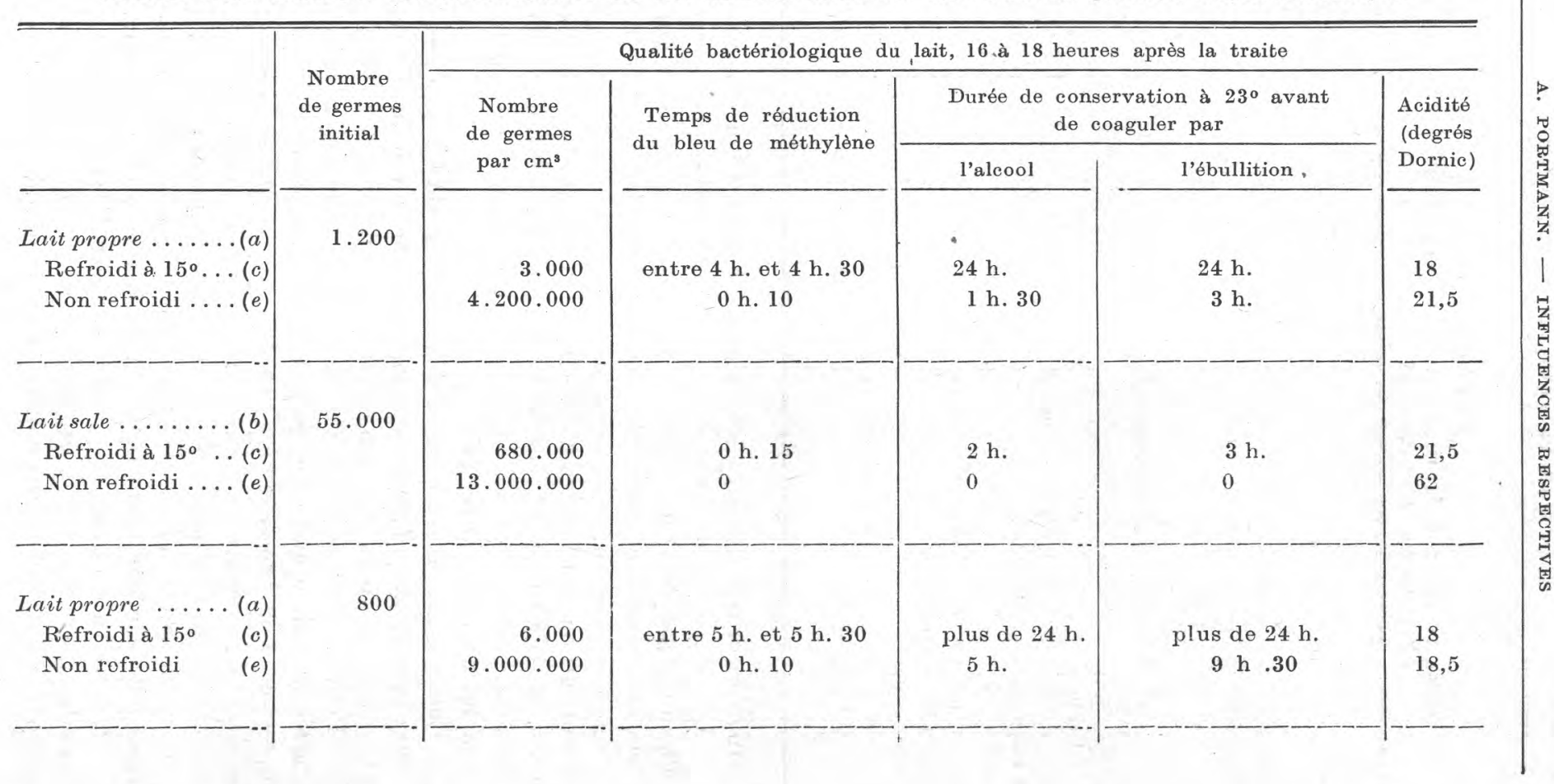




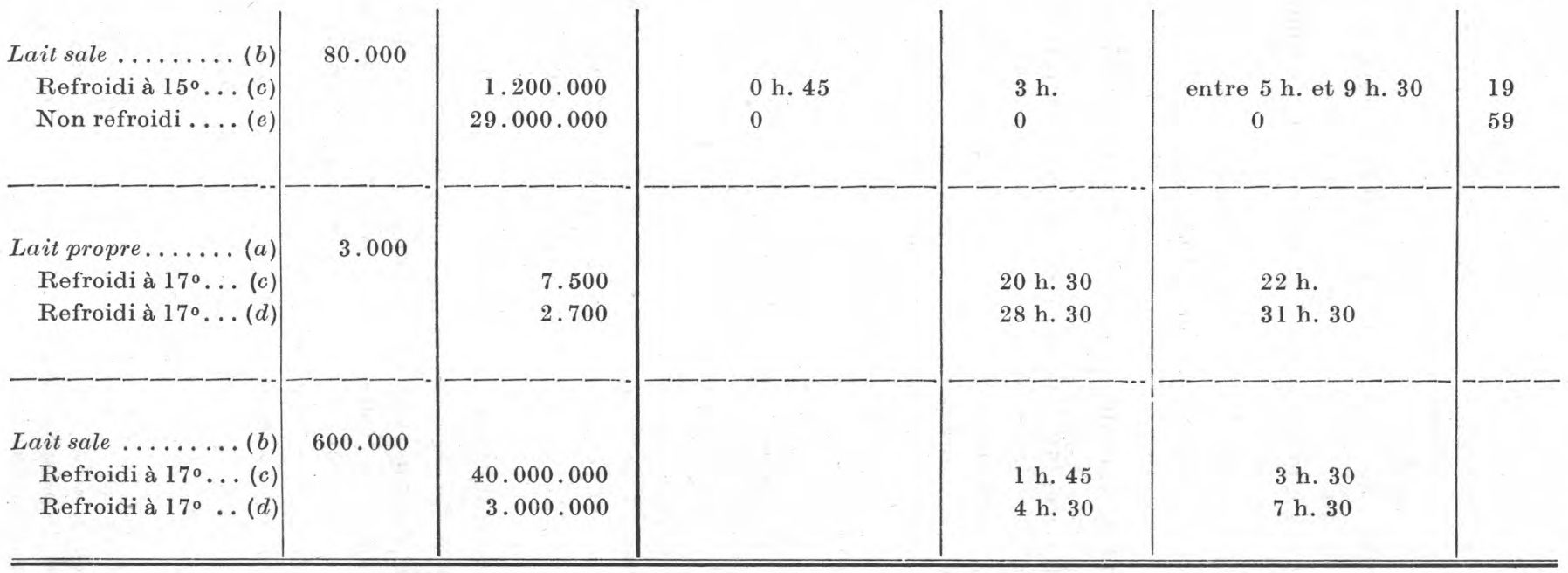

a) Lait propre : lait reçu dans des ustensiles de laiterie soigneusement nettoyés et stérilisés.

b) Lait sale : lait reçu dans des ustensiles ayant été nettoyés suivant l'habitude de l'exploitation.

c) Lait refroidi à $15^{\circ}$ ou $17^{\circ} \mathrm{immédiatement} \mathrm{après} \mathrm{la} \mathrm{traite} \mathrm{et} \mathrm{conservé} \mathrm{à} \mathrm{la} \mathrm{température} \mathrm{atmosphérique} \mathrm{pendant} 16$ à 18 heures

d) Lait refroidi à $17^{\circ}$ immédiatement après la traite et maintenu à $15^{\circ}$ pendant 18 heures.

e) Lait non refroidi et conservé à la température atmosphérique pendant 16 à 18 heures. 
améliorée pour que le lait soit susceptible d'être pasteurisé le lendemain, sans même avoir été refroidi après la traite : ce lait après avoir été maintenu 16 à 18 heures à la température atmosphérique possédait encore une durée de conservation de $1 \mathrm{~h}$. 20 à 5 heures. Le refroidissement à $15^{\circ} \mathrm{du}$ lait trait dans des ustensiles nettoyés sommairement, permettait de parvenir à un résultat sensiblement identique, mais ce même refroidissement prolongeait jusqu'à 24 heures la durée de conservation du lait trait dans du matériel soigneusement nettoyé.

Le lait trait dans des ustensiles nettoyés sommairement et non refroidi était, le lendemain, caillé dans le bidon.

\section{Refroidissement faisant appel au froid artificiel}

Plus la température du lait est abaissée et plus longtemps il se conserve. Toutefois, afin de ne pas descendre à une température inutilement basse, ce qui nécessiterait une dépense d'énergie excessive, il faut pouvoir déterminer la température maxima compatible avec une bonne conservation et étudier les différents procédés qui permettent d'y parvenir. L'essai suivant a eu pour but d'étudier ce problème (1).

Huit méthodes de refroidissement faisant appel au froid artificiel et quatre méthodes utilisant l'eau courante à température de $13-15^{\circ}$ ont été expérimentées; 300 litres de lait traits entre 17 et 19 heures ont été mélangés dans un bac stérilisé et répartis dans treize bidons préalablement stérilisés.

Le lait utilisé provenait de quatre fermes différentes choisies de façon à représenter la moyenne des exploitations de la région.

Les caractéristiques de la journée au point de vue météorologique étaient les suivantes :

Température atmosphérique moyenne .... $17^{\circ} 6$

Température maxima............. 24\%9

La qualité bactériologique, qui correspondait à celle que l'ón observe souvent pendant les jours chauds, était très mauvaise : 1 million de germes au centimètre cube, 2 heures environ après la traite, au moment où le lait était rassemblé à la laiterie.

Les méthodes de refroidissement utilisées étaient les suivantes :

a) Aspersion continue d'eau glacée alimentant un tourniquet hydraulique;

(1) Cet essai a été effectué sous la direction de $M$. Mocquot et avec la collaboration de M. Lesseur, Directeur de la Laiterie Coopérative de La Roche-Brienon et de M. Lablanchy, de la Laiterie Coopérative d'Auxerre. Les courbes de refroidissement ont déjà fait l'objet d'une publication par M. Lablanchy [3]. 
b) Bidon plongeant dans de l'eau glacée brassée par un agitateur;

c) Courant d'air froid ; le bidon était placé dans un petit tunnel installé dans une chambre frigorifique, un ventilateur soufflait de l'air réfrigéré à une extrémité du tunnel, le lait lui-même était agité, à l'intérieur du bidon, au moyen de l'hélîce d'un tourniquet hydraulique entraîné par un petit moteur hydraulique;

d) Courant d'air froid, même principe que ci-dessus, mais sans agitation du lait;

e) Bidon plongeant dans de l'eau glacée sans agitation ;

f) Tourniquet hydraulique, aspersion d'eau à $14^{\circ}$ jusqu'à abaissement de la température du lait à $16^{\circ}$, bidon placé ensuite dans un local à $0^{\circ}$;

g) Tourniquet hydraulique, aspersion d'eau à $14^{\circ}$ jusqu'à abaissement de la température du lait à $15^{\circ} 4$, bidon placé ensuite dans un local à $12^{\circ}$;

h) Bidon placé dans un local à $0^{\circ}$;

i) Tourniquet hydraulique, aspersion d'eau glacée jusqu'à abaissement de la température du lait à $1^{\circ} 4$, bidon placé ensuite dans un local à $25^{\circ}$;

j) Tourniquet hydraulique, aspersion d'eau à $14^{\circ}$ jusqu'à abaissement de la température du lait à $16^{\circ} 4$, bidon placé ensuite dans une bassine contenant de l'eau à $14-15^{\circ}$;

k) Tourniquet hydraulique, aspersion d'eau à $14^{\circ}$ jusqu'au moment du prélèvement des échantillons;

l) Tourniquet hydraulique, aspersion d'eau à $14^{\circ}$ jusqu'à abaissement de la température du lait à $15^{\circ} 4$, bidon placé ensuite dans un local à $25^{\circ}$;

$m$ ) Bidon témoin non refroidi et porté dans un local à $25^{\circ}$ dès le début de l'essai.

L'examen du tableau IX montre que six méthodes ( $a$ à $f$ ), nécessitant toutes un apport de froid artificiel, ont donné des résultats qui, compte tenu de la mauvaise qualité bactériologique initiale du lait, peuvent être considérés comme satisfaisants.

Les six autres méthodes $(g$ à $m$ ) bien que nécessitant l'une un local à $0^{\circ}(h)$ et l'autre de l'eau glacée $(i)$ sont insuffisantes.

Cette classification des méthodes n'a qu'une valeur de comparaison : si au lieu d'un lait contenant 1 million de germes par centimètre cube nous avions eu à notre disposition un lait moins contaminé certains des procédés reconnus comme insuffisants seraient devenus acceptables. 
TABLEAU IX

QUALITÉ BAGTÉRIOLOGIQUE D'UN LAIT REFROIDI PAR DIFFÉRENTES MÉTHODES

\begin{tabular}{|c|c|c|c|}
\hline & $\begin{array}{c}\text { Durée de conservation à } 23^{\circ} \\
\text { avant coagulation à l'ébullition }\end{array}$ & $\begin{array}{l}\text { Nombre de germes } \\
\text { par } \mathbf{c m}^{3}\end{array}$ & $\begin{array}{l}\text { Acidité } \\
\text { (degrés Dornic) }\end{array}$ \\
\hline $\begin{array}{l}\text { Lait du bac avant la répartition en bi- } \\
\text { dons } \ldots \ldots \ldots \ldots \ldots \ldots \ldots \ldots \ldots \ldots \ldots \ldots \ldots\end{array}$ & 9 h. 00 & 1.000 .000 & \\
\hline $\begin{array}{c}\text { Lait soumis à différentes méthodes de } \\
\text { refroidissement }\end{array}$ & \multicolumn{2}{|c|}{$\begin{array}{l}\text { (Echantillons prélevés } 13 \mathrm{~h} .30 \\
\text { après la répartition en bidons) }\end{array}$} & $\begin{array}{l}\text { (Echantillons prélevés } 17 \mathrm{~h} .30 \\
\text { après la répartition en bidons) }\end{array}$ \\
\hline a) Aspersion continue d'eau glacée .... & 8 h. 30 & 1.000 .000 & 19 \\
\hline b) Bidon plongé dans l'eau glacée agitée & 8 h. 30 & 1.200 .000 & 18,5 \\
\hline c) Courant d'air froid, lait agité ....... & 7 h. 00 & 1.100 .000 & 19 \\
\hline d) Courant d'air froid, lait non agité ... & 6 h. 30 & 1.200 .000 & 19 \\
\hline 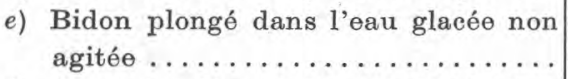 & 6 h. 30 & 1.000 .000 & 18,5 \\
\hline f) Aspersion d'eau à $14^{\circ}$ puis local à $0^{\circ}$. & $5 \mathrm{~h} .00$ & 1.000 .000 & 18,5 \\
\hline h) Local à $0^{\circ} \quad \ldots \ldots \ldots \ldots \ldots \ldots \ldots$ & 2 h. 30 & 5.400 .000 & 22 \\
\hline i) Aspersion d'eau glacée puis local à & & & \\
\hline $25^{\circ} \ldots \ldots \ldots \ldots \ldots \ldots \ldots \ldots \ldots \ldots \ldots \ldots \ldots \ldots \ldots$ & 1 h. 30 & 5.800 .000 & 27 \\
\hline j) Aspersion d'eau à $14^{\circ}$ puis immersion & & & \\
\hline dans l'eau à $14-15^{\circ} \ldots \ldots \ldots \ldots$ & $1 \mathrm{~h} .00$ & 17.000 .000 & 28 \\
\hline k) Aspersion continue d'eau à $14^{\circ} \ldots$. & $1 \mathrm{~h} .00$ & & 26,5 \\
\hline l) Aspersion d'eau à $14^{\circ}$ puis local à $25^{\circ}$ & 0 h. 00 & 21.000 .000 & 40 \\
\hline$m)$ Témoin à $25^{\circ} \ldots \ldots \ldots \ldots \ldots \ldots \ldots$ & $\begin{array}{c}\text { coagulé au moment du prélè- } \\
\text { vement des échantillons }\end{array}$ & & 76 \\
\hline
\end{tabular}




\section{GONCLUSION}

On sait qu'en abaissant la température du lait aux environs de $0^{\circ}$, on prolonge sa durée de conservation. Toutefois, l'action du froid se borne à arrêter le développement microbien, mais ne modifie pas la qualité bactériologique initiale du lait.

On obtient le maximum d'efficacité avec le minimum de frais en refroidissant le lait à une température moyennement basse, à laquelle le développement bactérien n'est que ralenti, à condition d'avoir soin d'éviter au maximum les contaminations au moment de la traite.

Il est difficile de préconiser une température de refroidissement optima : celle-ci devra varier en fonction de la qualité bactériologique initiale du lait et en fonction de la qualité bactériologique "finale" que l'on se propose d'obtenir au moment où le lait arrive à la laiterie : moins le lait sera pollué au moment de la récolte, moins il sera nécessaire d'abaisser sa température au-dessous de $15^{\circ}$.

Si l'effort se porte sur le nettoyage de la vaisselle laitière - et bien entendu sur la propreté de l'étable - la qualité bactériologique du lait sera suffisamment améliorée pour que l'utilisation d'un refroidissement modéré (environ $15^{\circ}$ ), effectué aussitôt après la traite, permette de maintenir cette qualité dans des limites acceptables, surtout si les bidons de lait sont ensuite entreposés dans une pièce fraîche ou le long d'un mur situé au nord, à l'abri du soleil jusqu'au passage du ramasseur.

En effet, comme le montre le tableau VIII, le lait trait dans du matériel soigneusement nettoyé contient dès la traite, 50 à 200 fois moins de germes que le lait trait sans précautions spéciales de propreté. Le refroidissement, suivi d'une conservation pendant 15 à 18 heures à la température atmosphérique, accentue encore cette différence entre les deux sortes de laits : le lait récolté proprement renfermant 200 à 5.000 fois moins de germes que l'autre.

Le refroidissement modéré du lait peut être obtenu par l'emploi du tourniquet hydraulique qui, suivant les possibilités apportées par l'installation d'eau à la ferme, abaisse la température du lait de 33 à $15^{\circ}$ en 15 à 20 minutes, la consommation d'eau restant sensiblement la même quel que soit le débit. Cette eau peut ultérieurement être utilisée pour abreuver les animaux, si l'on a pris soin de la recueillir dans un réservoir convenablement placé.

Tant que le lait produit à la ferme ne sera pas, aussitôt après la récolte, de bonne qualité bactériologique, les procédés de refroidissement à utiliser en vue d'obtenir pour ce lait une durée de conservation acceptable devront faire appel au froid artificiel : aspersion continue d'eau glacée en circuit fermé ou bidons placés 
dans de l'eau glacée agitée, ces opérations devant s'effectuer dès que possible après la traite; l'utilisation d'armoires frigorifiques à 2 ou $3^{\circ}$ dans lesquelles les bidons sont entreposés est insuffisante si la température du lait n'a pas été préalablement abaissée à $15^{\circ}$ dans les deux heures qui suivent la traite.

Ces procédés peuvent se concevoir dans l'installation de centres groupant le lait de tous les producteurs d'une commune mais leur application est plus difficile dans les fermes peu importantes.

Le producteur ignore très souvent que le lait qu'il récolte, sauf lorsque celui-ci coagule dans les bidons, ne présente pas une qualité bactériologique suffisante. Il ne sait pas non plus que les soins. apportés au nettoyage de la vaisselle laitière ont une aussi grande importance.

Il apparaît nécessaire, d'une part de le renseigner à ce sujet et ceci peut être fait très efficacement par des moniteurs envoyés par les laiteries - d'autre part d'attribuer une prime aux producteurs livrant du lait de très bonne qualité bactériologique, ce qui se traduit par un effet certain d'encouragement. Plusieurs usines se sont déjà engagées dans cette voie avec succès.

A la suite de trois concours de propreté du lait, effectués dans l'Yonne, en 1950-1951-1952 [4], il a été démontré que la classification des producteurs suivant la qualité bactériologique du lait livré était facile et pouvait se faire, par exemple, en utilisant des tests simples traduisant fidèlement la contamination initiale, tel que celui de la coagulation par l'alcool après incubation du lait à $23^{\circ}$.

On peut espérer que dans les régions où l'on réussira à établir entre les producteurs et les usines de transformation cette coopération efficace une amélioration sensible de la qualité bactériologique du lait sera obtenue.

(Nous exprimons notre profonde reconnaissance à $\mathrm{M}$. Mocquot. qui, en dirigeant notre travail, nous a permis de le mener à bien.)

\section{BIBLIOGRAPHIE}

[1] F. Dané. Bull. Techn. Inform. Min. Agr. 1951, 64, p. 587.

[2] A. Rowlands, H. Barkworth, Z. Hosking et O. Kempthorne. Journal. Dairy Res., 1950, 17, p. 159.

[3] A. Lablanchy. Rev. prat. Froid, avril 1954.

[4] G. Mocquot, J. Michet, R. Bigorre ot A. Portmann. Bull. Techn. Inform. Min. Agr. 1953, 77, p. 201. 